\title{
A Survey of Methods for Gas-Lift Optimization
}

\author{
Kashif Rashid, William Bailey, and Benoît Couët \\ Uncertainty, Risk \& Optimization, Schlumberger-Doll Research, Cambridge, MA 02139, USA \\ Correspondence should be addressed to Kashif Rashid, krashid@slb.com
}

Received 5 April 2012; Accepted 7 June 2012

Academic Editor: Azah Mohamed

Copyright () 2012 Kashif Rashid et al. This is an open access article distributed under the Creative Commons Attribution License, which permits unrestricted use, distribution, and reproduction in any medium, provided the original work is properly cited.

\begin{abstract}
This paper presents a survey of methods and techniques developed for the solution of the continuous gas-lift optimization problem over the last two decades. These range from isolated single-well analysis all the way to real-time multivariate optimization schemes encompassing all wells in a field. While some methods are clearly limited due to their neglect of treating the effects of interdependent wells with common flow lines, other methods are limited due to the efficacy and quality of the solution obtained when dealing with large-scale networks comprising hundreds of difficult to produce wells. The aim of this paper is to provide an insight into the approaches developed and to highlight the challenges that remain.
\end{abstract}

\section{Introduction}

The introduction of lift gas to a non-producing or lowproducing well is a common method of artificial lift. Natural gas is injected at high pressure from the casing into the wellbore and mixes with the produced fluids from the reservoir (see Figure 1). The continuous aeration process lowers the effective density and therefore the hydrostatic pressure of the fluid column, leading to a lower flowing bottom-hole pressure $\left(P_{\mathrm{bh}}\right)$. The increased pressure differential induced across the sand face from the in situ reservoir pressure $\left(P_{r}\right)$, given by $\left(P_{r}-P_{\mathrm{bh}}\right)$, assists in flowing the produced fluid to the surface. The method is easy to install, economically viable, robust, and effective over a large range of conditions, but does assume a steady supply of lift gas [1]. At a certain point, however, the benefit of increased production due to decreased static head pressure is overcome by the increase in frictional pressure loss from the large gas quantity present. This has the effect of increasing the bottom-hole pressure and lowering fluid production. Hence, each well has an optimal desirable gas-lift injection rate (GLIR). However, when the entire gathering network is considered, the optimal gas-lift injection rate differs from that which maximizes individual well production due to the back pressure effects (the pressure drop observed across flow lines due to common tie backs further downstream) imposed by connected wells further downstream.
As a field matures, the greater demand for lift gas in conjunction with limitations imposed by existing facilities and prevailing operating conditions (compression capacity, lift gas availability, well shut-in for workover, etc.) can prevent optimal production from being achieved. In the absence of all operating constraints, other than the available lift gas, it is necessary to optimally allocate the available lift gas amongst the gas-lifted wells so as to maximize the oil production. This is the most basic definition of the gaslift optimization problem and is equivalent to an optimal allocation problem. Consideration of additional operating constraints, choke control for well-rate management and the treatment of difficult to produce wells, gives rise to a broader problem definition. In general, either definition can additionally accommodate an economic objective function, by inclusion of production and injection cost factors. Although the choice of objective function has been stated as the differentiator between the methods developed by some [2], in actuality, most methods can handle either definition and should not be categorized on this basis.

It is worth noting that generally the gas-lift design problem, that is, deciding the gas-lift valve (GLV) number and their depths, is excluded from the allocation problem, largely because the well configuration is already complete by the production stage and is considered invariable. Additionally, lift gas injection normally takes place at the deepest valve at the available injection pressure, where the depths have 


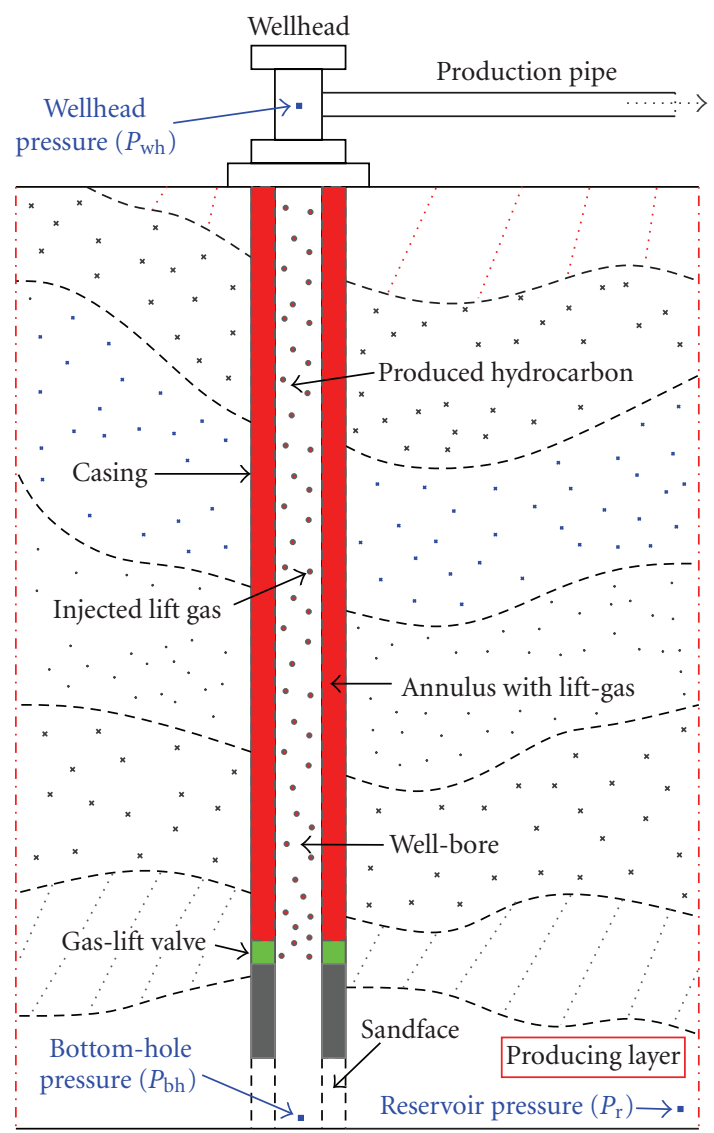

FIgURE 1: Gas Lift Well Schematic.

been set in advance. This does not preclude well redesign scenarios (workovers) that could yield better production results, whose optimization may improve. For example, Mantecon [3] focused on studies to increase oil production by redesigning individual wells using data gathered at the well site. Individual well performance improvement was considered a necessary requirement prior to field-wide optimization. This involved reevaluating depth of injection, GLIR, injection pressure, GLV size, packer installation and even changing the gas-lift type to intermittent (IGL) or plunger gas-lift (PL). The installation of packers enabled injection pressure increase and deeper GLV activation, collectively leading to increased production within facility handling limits. Although such improvements increase well stability and allow wells to produce more readily, the need for optimal allocation is not obviated. In fact, it is for the reason that the handling facilities and operating resources are constrained that an optimal allocation is required and is most effective when well operation is stable.

In the following sections, a review of methods and techniques developed for the gas-lift allocation problem is presented. These concern, in chronological order of presentation, the generation of gas-lift performance curves from well test data, single-well nodal analysis and sensitivity studies, pseudo steady-state curve-based models that neglect well interactions, steady-state solutions based on network simulators, coupled reservoir and surface facility simulation, ultimately, leading to a fully integrated asset modeling approach. For the benefit of the reader, the key developments are summarized in Table 1 together with the main advantages and disadvantages which will be discussed next.

\section{Well Gas-Lift Performance}

Actual well investigation concerns physical well tests conducted at the well site. Fluid composition, PVT and other related tests provide information about the conditions of the well and its potential productivity. In addition, steprate gas injection tests can provide an accurate description of the behavior of fluid production with increasing lift gas injection.

The nature of single-well testing naturally gave rise to the development of tools to model the behavior of a single well, given certain input parameters to define the completion, fluid composition, pressures, and temperatures, both at the wellhead and at the point of contact with the reservoir. These Nodal Analysis tools enable a computational model of the well to be defined, with either a simple black oil or a more detailed compositional fluid description, commencing from the sand face, through the perforations, from bottom hole, through tubing, to the wellhead and further downstream to a delivery sink [4]. This model can then be used to predict the behavior of multi-phase flow through the well (see Figure 1) and the more representative it is of the prevailing conditions the better. Thus, to overcome the complication of performing costly and time-consuming step-rate tests in a field with many wells, these tools can be used to provide lift performance curves for all lifted wells $[5,6]$. Clearly, the analysis of a single well provides an incomplete picture with respect to the entire field performance and especially for optimal gas-lift allocation. However, it serves two important purposes. Firstly, all network simulators, which couple the behavior of individual wells in a field by a common gathering network, are based on such singlewell models. That is, in solving a network solution, the underlying multi-phase fluid flow behavior is calculated using nodal analysis tools. Secondly, in the absence of actual step-rate test data, lift performance curves are estimated by running sensitivity models over each of the individual wells. It is these lift performance curves that are used in gas-lift optimization studies. This is undertaken correctly, when the effect of interdependent wells is accounted for by updated lift performance curves at new conditions, but incorrectly when the resulting pseudo steady-state solution is accepted after only one cycle. The latter is a common assumption in many papers, often for the sake of simplicity, if not by oversight. The complete, or final, steady-state solution is one derived from the network simulator in which a rigorous pressure balance is achieved over all nodes in the network after the allocation of lift gas has been made to the wells. This is necessary since the back pressure imposed by the injection of lift gas in one well will affect the production from all connected wells. Hence, for optimal production the lift gas must be suitably allocated whilst accounting for 
TABLE 1: Key developments. The evolution of approaches developed for the treatment of the gas-lift optimization problem.

\begin{tabular}{|c|c|}
\hline Merits & Limitations \\
\hline \multicolumn{2}{|c|}{ Performance curve generation } \\
\hline Provides well production & Well test requirements \\
\hline relationship with GLIR & Well test data quality \\
\hline \multicolumn{2}{|c|}{ Nodal analysis } \\
\hline Well model simulation & Fluid data assumptions \\
\hline Multi-phase flow modeling & $P$ and $T$ assumptions \\
\hline Performance curve generation & Primarily for single well \\
\hline \multicolumn{2}{|c|}{ Curve-based models } \\
\hline Fast, analytical models & Neglect well interations \\
\hline Considers all wells & Curve fitting and quality \\
\hline Simple to evaluate & Pseudo steady state solution \\
\hline \multicolumn{2}{|c|}{ Network simulation } \\
\hline Rigorous simulation models & Evaluation cost \\
\hline Includes well interactions & Model smoothness \\
\hline Handles looped models & Steady state solution \\
\hline Handles facility components & Gradient information \\
\hline \multicolumn{2}{|c|}{ Coupled simulation } \\
\hline Detailed coupled system & Coupling scheme \\
\hline Rigorous interaction & Model robustness \\
\hline \multirow[t]{2}{*}{ Includes transient effects } & High computation cost \\
\hline & Gradient information \\
\hline \multicolumn{2}{|c|}{ Integrated asset modeling } \\
\hline Comprehensive system & Coupling procedures \\
\hline dynamics and interactions & Model robustness \\
\hline \multirow[t]{2}{*}{ Simulation over asset life } & High computation cost \\
\hline & Gradient information \\
\hline
\end{tabular}

well interaction. Lastly, facility handling constraints (i.e., constraints imposed at a field-wide level, e.g., maximum water production, etc.) are also only applicable if the entire network model is considered.

\section{Single Well Analysis}

The use of nodal analysis to generate the gas lift performance curve of a single well based on actual pressure and temperature surveys along with a suitable multiphase flow correlation is well established [7-9]. The optimum GLIR is often simply set to that furnishing the highest production rate on the gaslift performance curve (GLPC) (see Figure 2). The maximum GLIR and maximum oil rate can be used to establish the optimal valve depth setting and the wellhead pressure $\left(P_{\text {wh }}\right)$ [9]. However, the single-well configuration, considered in isolation of other wells, is not a field gas-lift optimization solution.

A more accurate well model, based on mass, energy, and momentum balance, was proposed by Vazquez-Roman and Palafox-Hernandez [10]. A single-well case was examined to determine the injection depth, pressure, and the amount of gas injection, using a commercial optimization program based on a hybrid interior algorithm. The results were reported as being more accurate and therefore better suited for field-wide simulation studies, than the standard nodal approach. However, no results were reported for a fieldwide application. Note that the use of compositional models over simple black oil models is also recommended practice for better accuracy $[11,12]$. Indeed, the best possible well model, yielding representative gas-lift performance curves, is desirable for both simulation and optimization purposes.

Dutta-Roy and Kattapuram [13] investigated the introduction of lift gas to a single well using nodal analysis. The back pressure effects imposed by gas injection on two wells were considered, prior to an investigation of a 13 well network. It was noted that single-well methods were inadequate for analyzing a production network of many gaslifted wells and that a general network solver is thus required. That is, optimizing wells systematically, but on a wellby-well basis, will not guarantee that an optimal solution will be obtained for the entire network. Equally, Mantecon [3] noted that for field-wide optimization, accurate gas injection and liquid production estimates are desired, but more importantly, as the conditions in one well affect other connected wells, a computer simulation is required to effectively account for the interactions. This cannot be achieved manually.

Bergeron et al. [14] used step-rate well test data to obtain the characteristic lift performance curve for a single offshore well. A remotely actuated controller was used to manage the gas-lift injection rate in real time for production maximization, cost reduction and mitigation of blockages resulting from flow line freezing. Accurate flow rate and injection measurements were deemed necessary to ensure accurate model interpretation and optimization, while also ensuring stability from heading and slugging effects. The single-well optimization scheme adopted set the unconstrained optimum with an unlimited supply of lift gas or established the injection rate for production maximization in the presence of operating constraints, including the amount of lift gas available. Hydrate formation and low ambient temperatures, together with changes in lift gas supply, pressure, or quantity can lead to suboptimal levels of gas injection and consequently, a drop in cumulative well productivity. Continuous monitoring and sustained optimization were considered imperative to ensure that the well operated at maximum efficiency the majority of the time leading to an observable increase in total production. It was noted that to connect-up multiple wells, a more sophisticated optimization routine was necessary to handle the inter-related well allocation problem. Although only a single well was considered and the optimization scheme was limited to setting the lift gas rate resulting in the highest flow rate, the elements necessary for remote real-time field-wide gas-lift optimization are evident.

\section{Pseudo Steady-State Models}

In the previous discussion, it should be noted that direct step-rate well-site data provides a field model of sorts (case 1). Using PVT and composition data to generate gas-lift 


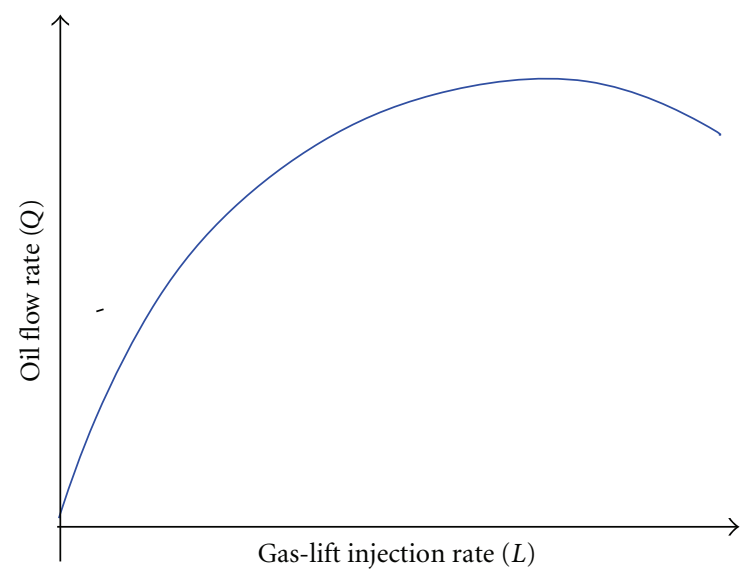

Figure 2: Gas Lift Performance Curve at a given Wellhead Pressure $\left(P_{\mathrm{wh}}\right)$.

performance curves using nodal analysis tools can provide independent single-well models (case 2). Thirdly, employing a rigorous multi-phase network simulator, a composite fullfield model is obtained (case 3). Whereas the latter implicitly models the back pressure effects imposed, leading to a steady-state solution, the first two approaches choose to neglect them. Hence, such methods (case 1 and case 2), though perhaps extendible, provide only a pseudo steadystate solution.

In the following, methods established for case 1 \& case 2 are considered as one, in that the descriptive well performance curves employed for any well may be obtained from a single-well model or exemplifying actual well behavior, directly from well-site step-rate tests. The clear limitation of case 1 and case 2 is that they do not account for the true back-pressure effects imposed by gas injection in one well on the other wells connected in the network. Although such methods result in pseudo steady-state solutions, unless explicitly stated, this limitation can be overcome by iterating on the procedure presented with updated well data and gaslift performance curves. In general, more detailed simulation models, including the reservoir and the process plant, can be defined with increasing complexity as case 4 and case 5, respectively. Note that the latter embodies the entire asset.

Simmons [15, 16], and Kanu et al. [17] describe the generation of well lift performance curves. Typically, tubing intake pressure as a function of the production rate is plotted for varying gas liquid ratios (GLR). The well inflow performance curve (IPR) is superimposed and the intersecting points with the tubing intake pressure curves identify the highest production possible for any given GLR. This data gives rise to the characteristic gas-lift performance curve (GLPC) for each well [18, 19] (see Figure 2). The most efficient injection rate was noted as the point at which the incremental revenue is equal to the incremental cost of injection and not simply when production is maximized [1518, 20].

Simmons [15, 16], Redden et al. [20], and Kanu et al. [17] accommodate economic factors by including the net gain (or profit) from oil and gas production, and the costs associated with gas compression, gas injection and water disposal, and so forth. For incremental profit as a function of gas injection Simmons $[15,16]$ considered the difference between the revenue and cost. The solution was defined at the point where the incremental profit is zero (see Figure $3(\mathrm{~d})$ ) and was referred to as the maximum daily operating cash income (OCI). Redden et al. [20] and Kanu et al. [17] similarly converted the GLPC into monetary units. Redden et al. [20] proposed the optimum point to occur when the slope of the revenue versus cost curve is one (see Figure 3(b)). Kanu et al. [17] referred to this solution on the GLPC as the economic slope (see Figure 3(a)) and the equilibrium between revenue and cost as the economic point. Under consistent revenue and cost factors, each of the aforementioned schemes gives rise to the same solution, including profit maximization as a function of gas injection at zero slope (see Figure 3(c)).

Kanu et al. [17] are often cited for the "concept" of the equal-slope solution. Note that the term "method" is not used here, as the method implemented to obtain that solution can vary. Moreover, the equal-slope concept arises only if the average economic slope is used based on averaged well properties (e.g., an average water fraction over all wells). In general, given the differing conditions of each well, each economic slope will differ. Kanu et al. [17] noted that, while the use of average properties simplifies the procedure, individual economic slopes should be used for increased accuracy. In actuality, the equal-slope concept features more palpably in the work of Redden et al. [20] (solving for unitary slope on the revenue versus cost curve; see Figure 3(b)) and even earlier in the work by Simmons $[15,16]$ (solving for zero incremental profit, see Figure 3(d)). Both employ the equal-slope concept, but as observed by Clegg [21], were not cited in the work of Kanu et al. [17, 22].

The reciprocal of the slope of the GLPC gives the incremental gas-oil-ratio (IGOR) [23]. This notion was used to define an optimal solution based on equal IGOR for each well, referred to as the marginal GOR (MGOR) by Weiss et al. [24] and is the same as the equal-slope concept. A proof of optimality for a two-variable model based on substitution is provided by Weiss et al. [24] and is stated to be inductively correct for higher dimensions. Notably, the optimal condition is based on satisfying the maximum gas capacity constraint. It overlooks the notion of an overabundance of available lift gas and therefore the likelihood of poor lift efficiency due to excessive lift gas utilization.

In recent work [25-27], the Newton Reduction Method (NRM) was presented as a means for optimal allocation. The method reduces the original problem to a solution of a composite residual function of one variable by treating the available lift gas as an equality constraint. The method is derived from a strict mathematical formulation and is provably optimal, assuming the GLPC are convex. The method is fast and returns an equal-slope based solution.

Simmons $[15,16]$ noted that GLIR for maximum daily operating cash income (OCI) (the point at which the incremental profit is zero, see Figure $3(\mathrm{~d})$ ) is lower than the GLIR for maximum oil production and the GLIR for maximum present value operating cash income (PVOCI) (when well reserves are also considered) is lower still. The 


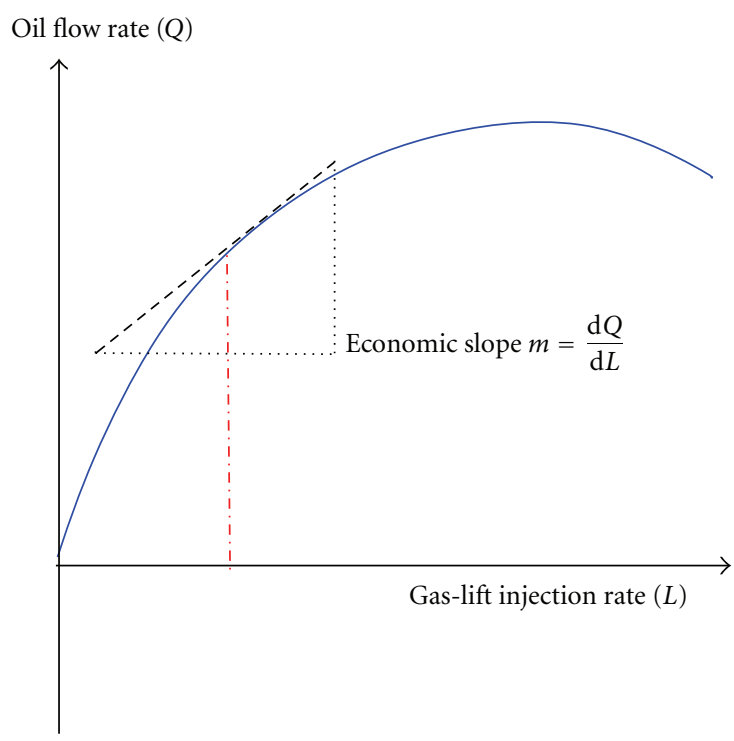

(a)

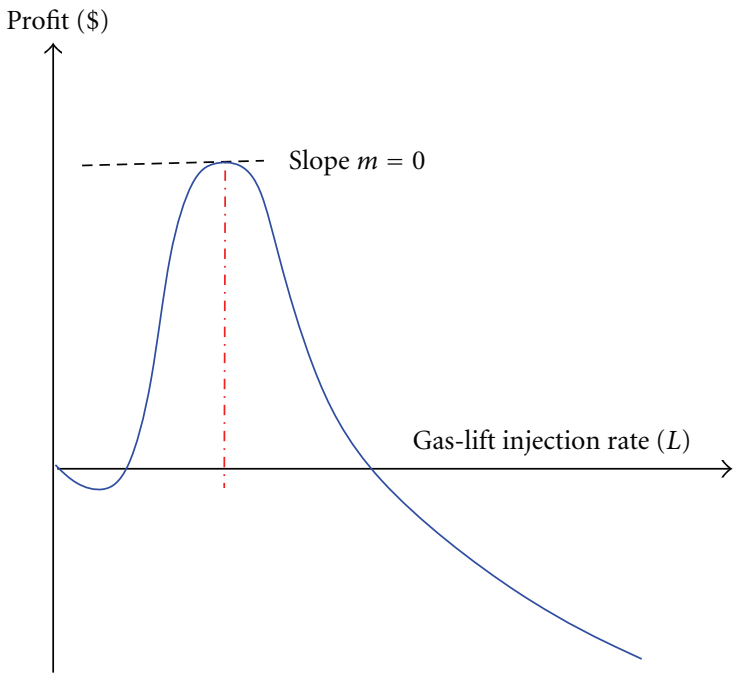

Loss $(\$)$

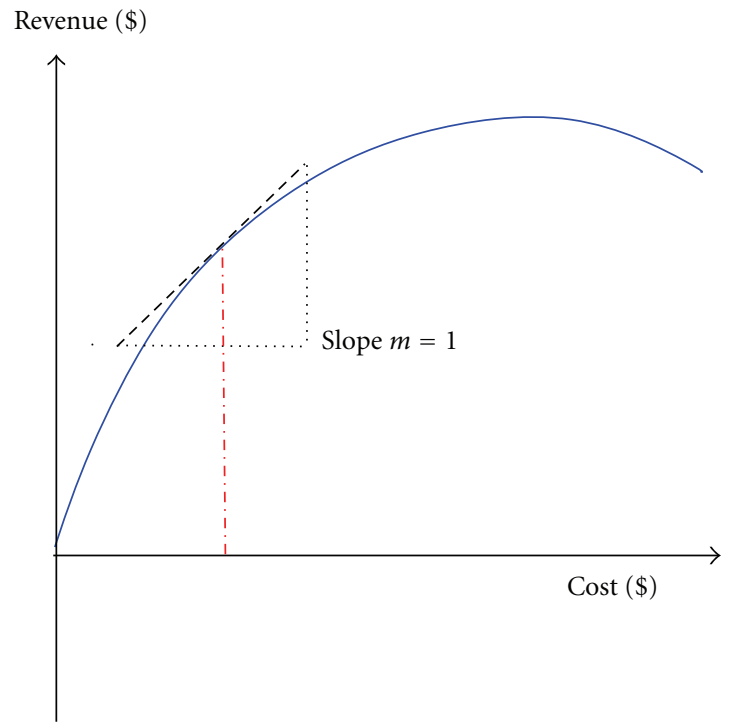

(b)

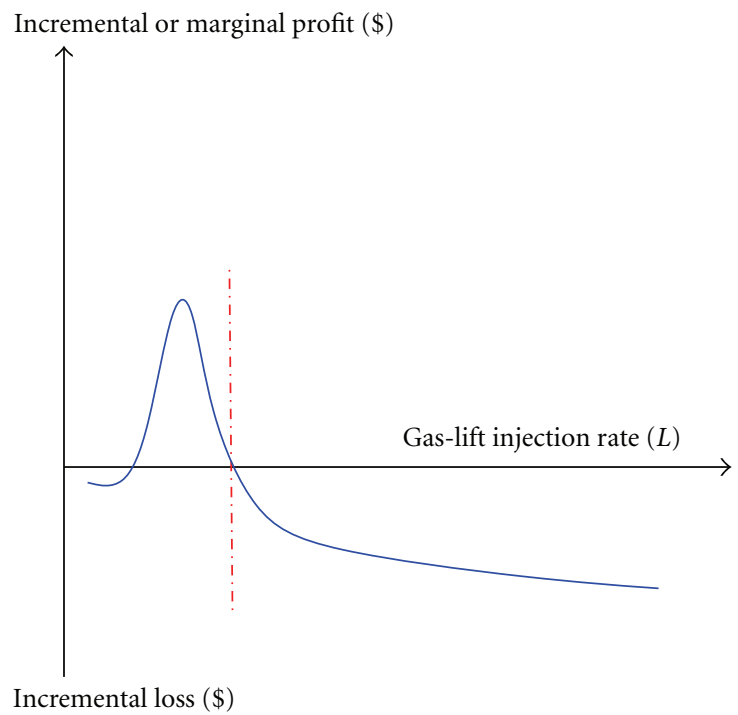

(d)

(c)

FIGURE 3: Performance curve representation and solution schemes.

latter (PVOCI) is expressed as the desired quantity, though it was observed that the PVOCI was insensitive to gas-lift rate variation above and below the optimum GLIR. However, exceeding the optimum GLIR causes facility overloading and yields lower PVOCI. The solution method is based on an incremental allocation of small units of gas until either maximum production, OCI or PVOCI are obtained. As the same slope is obtained for each well at the optimal point, this is an equal-slope based approach. It is interesting to note that many early implementations for gas-lift optimization adopted the same concept and a similar heuristic scheme [28, 29]. The gas-lift allocation procedure within the ECLIPSE reservoir simulator also uses a similar approach [30].

The multi-phase flow program and allocation procedure by Simmons $[15,16]$ proved useful in reducing the gas lift requirement to low producing wells when the gas supply was limited. A 20-well system was considered, however the predicted performance observed differed from reality due to a number of reasons. These included over-injection for well stability, misestimation of injection rates or wellhead pressures, inaccuracies in well data, correlation error, model parameter uncertainty and the heading problems observed. The last item concerns the effects of oscillatory fluctuation of pressure in a well. In general, these issues will confound any simulation-based approach and signifies the need for accurate data with which to develop the underlying models. As previously noted, a stable well configuration is desirable before performing optimization. Hence, overcoming heading for greater well stability may require well redesign. In this regard, Mantecon [3] and Everitt [31] both employed 
measures to identify and mitigate these issues (using redesign and intervention) prior to successful gas-lift allocation.

The Redden et al. [20] approach involves finding point at which the gradient of the monetary-based GLPC is one. However, no mention was made of the procedure to achieve this. If the lift gas allocation solution exceeds the available gas or separator capacity, a ranking of wells is made and the lift gas is removed from the lowest producing wells. The incremental reduction is continued until separator and compression limits have been met. This is not unlike the allocation scheme employed by Simmons $[15,16]$ however, in reverse. Such heuristic-based schemes are not proven to be optimal especially in the presence of multiple constraints and specifically in the given case where naturally flowing wells were excluded from receiving any lift gas at all. Results of a 10-well model were presented with and without capacity limitations in place.

Kanu et al. [17] solved for the economic point using a method derived graphically. The optimal operating condition is said to occur when the incremental revenue from production is equal to the incremental cost of injection in each well. The production and gas-lift rates for a range of slope values are estimated for each well. These give rise to slope versus production and slope versus gas-lift rate relationships. The economic point for each well is established and the associated lift-rate and production values are obtained. Total production and the total lift gas used are established by summing the individual well solutions. The same can be performed for all slope values, allowing the relationships of Total Production and Total Lift gas to be plotted with respect to the slope. With a limited supply of gas, the amount of gas available will indicate the expected slope value from the total lift gas versus slope plot. The associated production value can be obtained from the Total production versus slope plot for the given economic slope. Similarly, the individual well responses can be read from the particular well plots.

It is worth noting that the economic slope solution is greater than the zero gradient necessary for maximal production, which indicates the benefit of optimizing for economic performance and not simply production. A 6-well model was presented by Kanu et al. [17] with an unlimited lift gas supply using actual and an average estimate of the well properties. While the latter simplifies the evaluation process, the solution is less accurate. The constrained lift gas solution implicitly returns an average economic slope, leading to an allocation that is not strictly correct or optimal. In general, the procedure cannot easily handle additional constraints and can prove unwieldy for high-dimensional problems and cases where the curves have to be regenerated frequently due to changing well conditions. For this, the authors note that an automated procedure is necessary.

The equal-slope concept has been adopted in several other works [28, 31-33]. Typically, and incorrectly as the back pressure effects are neglected, when there is an unlimited supply of lift gas, the wells are often set to the GLIR that simply maximizes production in each well. With a limited supply, the equal-slope concept is employed.
Edwards et al. [32] fit each GLPC generated from a multi-phase flow simulator with a polynomial and identified the zero-gradient point of each well as the unconstrained optimum. In the limited case, an equal-slope solution was obtained using an iterative scheme, of which no particular implementation details were provided. Results for an 8-well model were presented.

Ferrer and Maggiolo [33] discussed the use of two computer models. The first, to interpret well behavior, performs diagnostics and well redesign. The second, to generate well relationships (GLPC) and perform optimization. The equalslope concept is employed if the gas supply is limited, however, only a single-well model was evaluated.

Everitt [31] used actual data to define the lift performance curve for each well. Although this can be costly and time-consuming, in comparison to simulation-based relationships, the curves obtained were representative of actual field conditions observed. The work focused on individual well performance identification and presented measures to alleviate the heading problems observed in several wells. That is, the oscillatory well fluctuations that occur due to changing pressure conditions in a well. The decision to close high water-cut wells helped reduce compression demand and cost.

Schmidt et al. [28] also employed the equal-slope concept for allocating lift gas, but details of the method were not provided.

The gas-lift optimization method cited by Chia and Hussain [29] is also based on the equal-slope concept. However, instead of simply using lift curve data, a simplified black oil network tool (GOAL) [34], employing a family of lift performance curves to describe the behavior of each of the wells, was used. A fast flow rate and pressure balance calculation enabled the back-pressure effects between connected wells to be accounted for during the allocation process. The available lift gas is discretized and allocated incrementally to the wells with favorable production gradients until an equalslope solution is obtained. The method is fast, but has the disadvantage of simplifying the fluid compositions of the wells, leading to some loss of accuracy in the solution.

The allocation of lift gas to a number of central processing facilities was considered by Stoisits et al. [23]. The composite lift performance curve was presented as the sum of the individual GLPCs for each of the wells connected to them. The MGOR concept, equivalent to the equal-slope solution, was used to allocate the available lift gas, but implementation details were not provided. A neural-network model was employed to simulate the behavior of the surface line hydraulics.

Weiss et al. [24] also employed the MGOR concept, in conjunction with developing a dedicated gas-lift allocation correlation based on empirical data. This provides the optimal GLR as a function of particular well properties, but treats only a single well in isolation.

Stoisits et al. [35] compared the empirically derived correlation based on a log equation from Weiss et al. [24] with the adaptive non-linear neural network model from Stoisits et al. [23]. The reported results differed markedly, possibly due to the difference in solution from the nodal analysis based runs used to train the neural network and the 
empirical data used to build the correlation. The latter is also potentially less versatile over a large range of conditions, as it conditioned to the well data employed.

Nishikiori et al. [2] presented the application of more standard non-linear optimization procedures for the gas-lift optimization problem. A first-order quasi-Newton method was developed. This is known to present super-linear convergence at a starting point close to the optimal solution. For this reason, three methods for automatically providing more informed GLIR starting points were considered. These include uniform lift gas distribution and the ratio of either the well productivity index (PI) or the maximum liquid production, to the corresponding sum for all lifted wells. The requirement of accurate first derivatives necessitated a costly central-difference scheme. A 13-well system was tested with both limited and unlimited gas availability. The method had the advantage of being fast and efficient.

Mayo et al. [36] used the Lagrange multiplier approach with each of the gas-lift performance curves modeled using second-order polynomials. The formulation gave rise to a convex constraint set and the imposition of Karush-KuhnTucker (KKT) conditions for optimality guaranteed the resulting solution as being globally optimal $[37,38]$. The method of solution was fast, though perhaps with some loss of accuracy due to the fitted polynomial, and was used to remotely actuate all 34 gas-lifted well valves through a controller and not just for a single-well as performed by Bergeron et al. [14]. In the unlimited case, the apex point of each GLPC, identifying maximal production, was set and for the limited case, the lift gas deficit (from the optimal lift gas requirement) was shared equally among the wells.

Lo [39] also used a Lagrangian formulation, but with the intent of dealing with multiple constraints. A general non-linear optimizer was employed (of which no details were given) to solve the constrained allocation problem. With a lift gas inequality constraint only, the equal MGOR solution was obtained for production maximization. In the presence of additional production constraints, the MGOR was noted to differ for each of the 20 wells in the model tested. Lo observed, with some foresight, that if, as proposed, the lift performance curves are assumed concave, then the objective function is concave and the feasible region defined by the constraints is convex. Thus, "one possibility for future work... is to devise a faster algorithm to solve the well management problem compared to [the] general nonlinear program" employed. In addition, as the back-pressure effects were not considered, the problem should be "solved iteratively until the predicted WHP are consistent." Both these features happen to be the basis behind the gas-lift optimization scheme encompassing the Newton Reduction Method (NRM) [27]. However, while the general non-linear scheme adopted by Lo [39] enables the application of many constraints, including those at the manifold level, the NRM approach is somewhat limited [25].

Fang and Lo [40] and Handley-Schachler et al. [41] both proposed the use of Sequential Linear Programming (SLP) techniques. The lift performance curves were assumed to be piece-wise linear and the constraints were linearized using a first-order Taylor series expansion. The approach can be fast, as it requires only first derivatives, and is able to handle bigger models with relative ease. However, the limitation is that the linear model maybe a poor representation of the highly nonlinear system and non-instantaneously flowing (NIF) wells can be problematic (see Figure 4). Both papers utilize separable programming, the adjacency condition and special order sets in order to qualify the lift performance curve of each well and to optimize the performance of the linear solver [40, 41].

Handley-Schachler et al. [41] presented a 21-well model for a de-bottlenecking study and a 250-well model for produced gas usage optimization problem, including the amount used as injection gas. The objective was to maximize revenue by lift gas allocation, while controlling other factors such as, compressor power, suction and discharge pressures. Fang and Lo [40] examined two full-field test cases, taking account of changing reservoir conditions, to maximize the cumulative oil production.

An improved procedure to generate the GLPC to more accurately match the test data collected was reported by Salazar-Mendoza [42]. On the other hand, Alarcón et al. [19] employed a modified second-order polynomial to fit the well GLPCs obtained from nodal analysis. The addition of a correction term gave rise to a better fit than the use of standard second-order polynomials reported by Nishikiori et al. [2] and Mayo et al. [36]. The standard non-linear problem was posed and the convexity of the curves noted to yield a globally optimal solution. The Sequential Quadratic Programming (SQP) method was used for the solution of the problem, using an approximate Hessian update procedure.

The SQP method is the workhorse of constrained nonlinear optimization. A quadratic approximation is made at a point on the Lagrangian function and the constraints are linearized. The resulting quadratic subproblem is solved for the direction of search, along which a line search is performed to minimize the merit function $[37,38]$. The matrix of second-order derivatives, the Hessian, must be updated to improve the quadratic approximation of the Lagrangian function using a suitable update procedure, for example, the BFGS method [38]. The cycle is repeated iteratively until convergence to a local minimum. The method suffers from the requirement of second-order derivatives, which can be costly and difficult to evaluate. The method can handle constraints well and from a good starting point can exhibit quadratic convergence. However, using approximate Hessian update procedures, only super-linear convergence can be guaranteed [37].

The need for a good GLIR starting condition was addressed by Alarcón et al. with a distribution of available lift gas proportional to the maximum flow rate over the sum of all wells, as also suggested by Nishikiori et al. [2]. For non-instantaneous flow (NIF) wells (i.e., those requiring a minimum level of gas injection before they can flow, see Figure 4), minimum GLIR rates were specified. This has the effect of forcing the wells to produce, but can lead to a suboptimal solution as certain wells unnecessarily consume large amounts of lift gas simply to produce small amounts of oil. Lo [39] noted that NIF wells break the concavity assumption and incorrectly suggested that as long as the minimum 


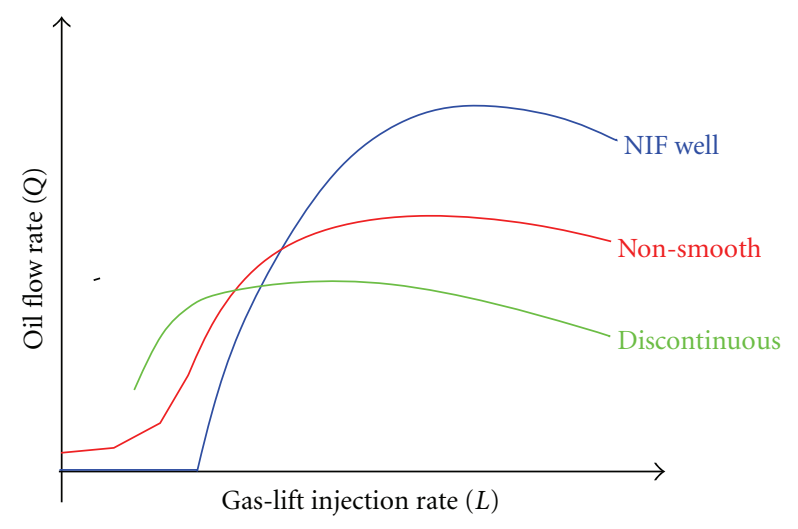

Figure 4: Gas Lift Performance Curves at a given Wellhead Pressure $\left(P_{\mathrm{wh}}\right)$.

GLIR is far from the GLIR required for the unconstrained maximum flowrate, the use of minimum GLIR constraints is reasonable. However the excessive allocation of lift gas to low producing wells that results, prevents better solutions from being obtained.

Alarcón et al. examined test cases of 5, 6, and 13-wells, of which the first two were also considered by Buitrago et al. [43] using a global derivative-free method. In addition, the 6-well problem was addressed by Ray and Sarker [44] using a multiobjective evolutionary approach. However, [19] only compared results from Buitrago et al. [43], from which the test cases were taken. The solutions from Alarcón et al. differed to those from Buitrago et al. [43] somewhat due to the method of solution employed and the curve interpolation scheme used. In general, the improved polynomial fit and the robust SQP solver yielded better results for Alarcón et al. Note, however, that the single NIF well present in the 6-well case was treated in a binary manner, that is, either on or off. The necessary treatment of a problem with a greater number of NIF wells was not made clear.

The complications arising from wells with nonsmooth gas-lift performance curves, discontinuities and indeed with non-instantaneous flow can impede the progress of conventional gradient-based optimization methods (see Figure 4). This results in sub-optimal solution due the poor handling of such wells and due to simplification made by curve fitting. For example, the flat section of a NIF well returns a gradient that is of little use in the optimization procedure. Hence, a minimum lift gas injection constraint is often implemented to overcome this issue, but this leads to an inefficient allocation and a sub-optimal solution, especially with increasing dimensionality. Conventional methods also tend to find only local solutions. To overcome these concerns, several researchers resorted to the use of more robust global search algorithms. Buitrago et al. [43] developed a global derivative-free search algorithm for the gas-lift allocation problem using heuristic measures to evaluate the descent direction. The method explores promising areas of the search space using a predefined number of samples using random search and a clustering method. The method is reported as being able to handle any number of wells, including wells with irregular profiles and non-instantaneous flow (NIF) (see Figure 4). The paper presented test cases of 5, 6, and 56 wells. The last two cases comprised 1 and 10 NIF wells, respectively. The method is compared to solutions obtained using an equal-slope approach. It is interesting to note that the solutions of the reported method by Buitrago et al. [43] are with the NIF wells turned off. However, a comparative study has shown that better solutions are possible with certain NIF wells activated [27]. Hence, although the method is robust, it does not appear to show any advantage in being able to accommodate NIF wells at all.

Buitrago et al. [43] proposed the metric of gas lift per barrel of oil at the solution point as a means of comparing solutions for different methods. However, while certain methods allocate all the available lift gas and find only local solutions, others will not. As such, the best comparative metric can only be solution quality, based on the overall objective value (e.g., the total oil production) in the absence of the computational cost necessary to obtain it. Note that many papers provide no function call count as a measure of computational cost or the efficiency of the method presented with increasing dimensionality. Lastly, it should be noted that for the third test case in Buitrago et al. [43], the sum of allocated lift gas is mis-stated. This should be 20,479 MSCF/D for the reported method and 24,661 MSCF/D, which exceeds the quantity of available gas, for the equalslope solution used for comparison. This changes the metric ratios reported from 0.939 and 1.059 , to 0.94 and 1.16, respectively.

The use of a genetic algorithm (GA) for the gaslift allocation problem for production maximization was reported by Martinez et al. [45]. A genetic algorithm is a stochastic population based global search strategy in which an initial set of candidate seeds, often randomly selected, is evolved over a number of generations using the key operations of crossover, reproduction, and mutation. The fittest candidate in the final gene pool is the solution to the optimization problem. The method is derivative-free, robust and can potentially find a global solution. In addition, both integer and continuous variables can be handled with ease. However, the method has the disadvantage of requiring a great number of function evaluations, which can be computationally expensive. Many variations and extensions exist to speed up convergence, maintain population diversity and to avoid binary string encoding. Algorithm parameter tuning may also be necessary for optimal performance. For example, in Martinez et al. [45], a greedy step was included to prioritize wells for preferential gas lift allocation and constant lift curves were used to test models of 10 and 25 wells. In addition, uniform and average crossover, mutation, shortand long-creep steps were considered for algorithm performance improvement. The method reportedly outperformed individual well schemes for production maximization by twenty percent.

Ray and Sarker [44] posed the gas-lift optimization problem as a multi-objective problem (MOP), with the intent to maximize production while minimizing lift gas usage. In the single objective problem of maximizing production alone, the second objective is treated as a constraint. 
A variant of the NSGA-II algorithm [46] was employed as a means of maintaining population diversity. The acronym refers to the nondominated sorting genetic algorithm, which indicates that the best solutions identifying the Pareto front are retained [46]. Contrary to Martinez et al. [45], where the robustness of the GA was used to help overcome the irregularities observed in the GLPCs, the lift curves were instead assumed to be piece-wise linear. The 6- and 56well cases from Buitrago et al. [43] were employed for testing. The derivative-free algorithm was robust and able to deliver solutions to the problems, each with 1 and 10 NIF wells, respectively. They reported improvements compared to the results by Buitrago et al. [43]. However, it should be observed that the simulations were run 96 times each and the best result obtained was used for comparative purposes. If the median or the average result is employed instead, the improvement is eroded or overcome completely.

The use of the MOP solution set to derive a production versus total gas injected profile in Ray and Sarker [44] is useful. However, retention of the solution configuration at each point on the profile is necessary, and no mention is made of this. In general, the MOP solution set is expensive and unnecessary given that much effort is required simply to filter out the dominated set of points. For example, the Newton Reduction Method will return the production versus total gas injected profile at selected values of the total available lift gas directly [25]. The benefit of multi-objective optimization could have been explored with more complex and conflicting operating constraints.

The shortcoming of curve-based network models, while better than undertaking single-well analysis, is evident in their neglect of the back pressure effects imposed by the entire network. The injection of lift gas in one will have an effect on all connected wells and this is not treated when the wells are considered separable. In addition, significant changes in well condition, bottom-hole pressure, productivity, water-cut or well-head pressure, all necessitate the update of the lift performance curves. As gas injection tends to lower BHP and increase WHP due to the fixed delivery point pressure, performance curve update is imperative. Hence, curve-based models give rise to pseudo steady-state solutions only and these will suffer from significant fluctuation in either well, operating or facility conditions, and with increasing model dimensionality. In the following section, network models are utilized to overcome this shortcoming.

\section{Network-Based Solutions}

The use of a non-linear constrained optimizer was reported as a necessity by Dutta-Roy et al. [47] when considering fields with many interconnected gas-lifted wells and facility components. A rigorous multi-phase flow network solver was used to solve the pressure and flow rates across the network in conjunction with a SQP solver for the constrained allocation problem. Function evaluation count was not reported, but the benefits of a simultaneous solution for more accurate results was shown $[13,47]$.

Nadar et al. [48] defined a network system to fully account for the production and gas-lift system interaction simultaneously for a complex offshore gas-lifted operation for production gain and cost reduction. A family of lift performance curves for varying WHP was generated for each well. The surface gas-lift components were modeled in the network simulator, including a detailed compressor model. The curves were assumed piece-wise linear and the SLP method employed for optimization. The separator pressure, gas-lift header pressure and the gas-lift injection rates were assigned as control variables. The approach was tested on 2 configurations of a composite model based on 4 fields, comprising 40 production platforms and 200 wells, for revenue maximization under equipment and network constraints. The method is able to handle large complex looped networks and can treat constraints simultaneously. A number of studies were examined, including gas-lift compressor train shutdown, establishing the gas-lift injection pressure and evaluating the gas transfer across fields. The difficulty of defining the gas-lift performance curves at low injection rates was noted, and thus can affect the solution quality. In general, inclusion of the gas compression and gas injection system enhances the overall merit of the optimization solution, in comparison to the production pipeline model alone.

Vazquez et al. [49] proposed an approach combining a genetic algorithm with the Tabu Search method. The latter performs a local search and retains a set of search points marked as in viable, known as the tabu steps. The search progresses in a direction where the objective function improvement is most likely. The method was tested on a 25well system and a 5\% increase in production was reported compared to the original state. The system considered comprised the production wells, the surface facility model and included a number of operating constraints. Although other forms of artificial lift (sucker rod pump and electric submersible pumps) were considered, the scheme can equally be applied to a gas-lift scenario. The main drawback of the approach was the high function evaluation cost resulting from the global stochastic-based scheme.

To ease the computational burden of simulation cost, Stoisits et al. [50] introduced an adaptive non-linear model to replace the actual network production simulator. The production model comprised the individual wells, surface line hydraulics model, and the production facility model. A GA was combined with a Neural Net (NN) based production simulation model for production optimization. The GA was used to return a global solution, while the NN model provided a fast proxy of the actual objective function once successfully trained. The problem investigated concerned the allocation of wells to production facilities and the lift gas to the wells, under multiple production constraints. The GA was used to find the optimal well activation state and the optimal drill site IGOR (for a collection of wells), which returns the GLIR for each individual producing well. Results from the GA were compared with and without parameter tuning and a production increase between $3 \%$ to $9 \%$ was achieved. In Stoisits et al. [35], the NN model was compared to the empirical correlation by Weiss et al. [24] using the composite IGOR curve definition developed [23]. 
An iterative offline-online procedure for gas-lift optimization was developed by Rashid [26, 27]. Gas-lift performance curves are extracted and used to solve the gas-lift allocation problem offline. Contrary to the assertion that spline-fitted GLPCs are unsuitable [19], the method uses smoothing and splines to ensure convexity of both the GLPC and the inverse derivative profiles required by the NRM solver (see Figure 5). The offline solution is passed to the network model [5] for updated wellhead pressures (WHP). The procedure repeats until convergence on WHP has been achieved [27]. This approach has the benefit of speed through the use of well curves, while retaining the rigor of the actual network model. Hence, flow interactions are fully accounted for. Note that the NRM solves a residual equation of one variable assuming GLPC convexity and a strict allocation of all available lift gas [27]. However, a GA solver is also embodied to handle nonconvex cases and the imposition of manifold level constraints that NRM is unable to handle with ease [25]. The method is particularly effective for managing high dimensional cases [51], including noninstantaneous flow wells and has also been extended to treat both gas-lift and choke control in each well using a mixed-integer formulation [52].

Kosmidis et al. [53] presented a mixed-integer non-linear program (MINLP) formulation for simultaneously treating well rates and GLIR for production optimization over multiple constraints using an iterative mixed-integer linear programming (MILP) scheme. The integer variables determined well activation status and the continuous variables the amount of lift gas received by each well. The well curves were assumed to be piece-wise linear. The method accounted for the well interactions and was reported as being able to handle non-smooth and non-instantaneous flow wells. A detailed production model was employed based on a black oil reservoir model (using the Peaceman inflow performance relationship [54]), a multi-phase well-bore model, a choke model [55], and a facility gathering model [53]. The MILP approach was defined using separable programming, special ordered sets, and the adjacency assumption, similar to the formulation adopted by Handley-Schachler et al. [41] for the SLP approach. The CPLEX solver from ILOG was used for the solution of the MILP sub-problem [56]. Two test cases were examined comprising 10 naturally flowing wells and 13 gas-lifted wells. The simplification arising from linear assumptions, both for the objective function and the constraints, can be a limitation of such an approach. However, results compared favorably to traditional heuristic approaches.

Camponogara and Nakashima [57] used a dynamicprogramming-(DP-) based formulation for the well-rate and lift gas allocation problem. The problem of determining which wells to produce and how much gas to allocate is a MINLP problem (as previously considered by Kosmidis et al. [53] and more recently, in Rashid et al. [52]). However, the DP algorithm solves the discretized gas-lift optimization problem approximately using precedence constraints to determine the activation of wells. Results were presented for a number of cases varying from 6 to 48 wells. The DP algorithm is relatively fast and despite the approximate

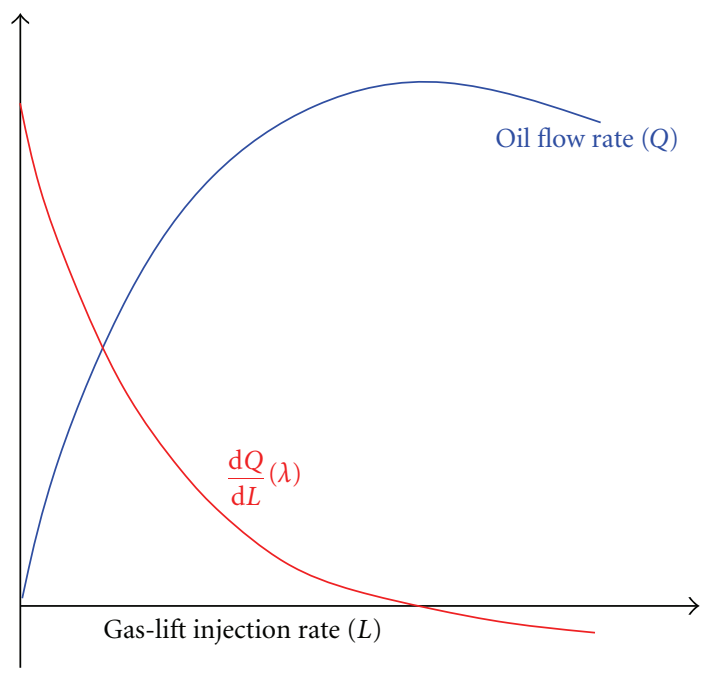

Figure 5: Lift Performance Curve and Derivative Profile.

nature of the approach, provides near-optimal solution for midsized networks (10-20 wells) with a sufficiently large model discretization. In general, the proposed formulation is NP-hard (this refers to non-deterministic polynomial time hard algorithm complexity) which indicates that the problem cannot be solved efficiently with increasing dimensionality due to the complexity arising from the increasing size of the connectivity graph.

The MINLP formulation for well-rate and lift gas allocation in Kosmidis et al. [53] was extended to accommodate the well scheduling problem [58]. The connection of wells to particular manifolds was treated simultaneously with wellrate management and lift gas allocation. The local solution method was deemed better than rule-based schemes, namely, those employing rules to rank and select well activation. However, the complexity of solving a large composite model for a local solution cannot guarantee a good solution overall unless executed from a good starting point or, at a higher cost, from many starting points.

\section{Network- and Reservoir-Based Solutions}

Davidson and Beckner [59] used the SQP method for wellrate allocation with a reservoir simulator. A simple reservoir with 3 production wells was considered with specified production target rates.

Wang et al. [60] used the SQP solver to simultaneously solve the well-rate and lift gas allocation problem for production optimization under many constraints, including minimum and maximum flow rates, pressure limits on wells or nodes, along with available lift gas and water production limits. The method was compared to an earlier MILP plus GA-based approach by the same authors [60]. The MILP formulation excluded the back pressure effect by arbitrarily estimating the WHP in building the gas-lift performance curve. A deliverability constraint is included in the revised approach to help determine the activation state of wells and to account for the flow interaction between them. Networks 
of 2,10 , and 50 wells were compared with water only, water and gas, or no constraints imposed. The SQP solver was shown to be fast and robust. The MILP approach, like other simplified approaches, ignored the effect of coupling and the constraints imposed by the network. As a downside, the proposed SQP-based method does not reportedly work with looped networks due to the greater potential of flip-flopping in the solution process.

The performance of three types of solver were compared by Fujii and Horne [61] for general application to a system for production optimization: a Newton-based method, a GA, and a polytope method. The latter is a derivative-free method that uses a fixed set of points describing a polytope in the search space. The points undergo operations of expansion, contraction and reflection in pursuit of a local optimum. The method is alternatively known as the Downhill Simplex method by Nelder and Meade [62]. Two test cases (of 2 and 10 wells) were considered, with the tubing size in each well as the decision variable, although the lift gas rate could equally have been used. The polytope was effective for small problems and the GA was effective at finding the global solution, even with greater number of variables present. The quasi-Newton method became trapped in local minimum and required costly derivative evaluations. For these reasons, the method proved less suited to problems with discontinuous objective functions (see Figure 4) and comprising many variables.

Carroll and Horne [63] compared a modified Newton method with the Polytope method. A reservoir component was included in the model and an economic cost function based on present value defined. A two-variable model comprising tubing size and separator pressure was considered. The benefits of simultaneous multivariate optimization were shown over a time-stepped simulation with changing reservoir conditions. The non-smoothness of the observed noisy function can complicate algorithm convergence and the finite-difference-based derivative evaluation scheme. The second-order Newton method required evaluation of both Jacobian and Hessian matrices. In order to obtain meaningful derivatives, a large finite-difference step was suggested. However, this can affect both the performance and quality of the solution. In general, the derivative-free polytope method proved more efficient and successful at finding good solutions. Performance metrics, in the form of objective function evaluation or time cost, however, were not provided.

Palke and Horne [64] considered the application of Newton, GA and Polytope algorithms in order to optimize $\mathrm{NPV}$ as a function of the well-bore configuration. Control variables included tubing diameter, separator pressure, choke diameter and the depth and rate of lift gas injection. The coupled simulation considered a reservoir with a single gaslifted well comprising reservoir, well, choke and separator component models. The Newton method (with Marquardt extension), the polytope method and the GA were compared using three problems. The first two problems concerned the tubing diameter and separator pressure, like Carroll and Horne [63], with two different fluid compositions in the well. As Fujii and Horne [61] and Carroll and Horne
[63] also concluded, the use of numerical derivatives over a non-smooth surface complicates the application of gradientbased methods. The results from the Newton method were expensive and poor. The third problem examined the variation of tubing diameter and lift gas rate over 4 time steps. The polytope method did not always converge and could not easily handle problems of many variables. The GA was robust and stable but required a great number of function evaluations at significant time and computational cost. Parameter tuning was attempted to increase convergence efficiency by varying mutation rate, changing the crossover scheme, employing fitness scaling and undertaking population culling [64].

Hepguler et al. [65], Kosmala et al. [66] and Wang and Litvak [67] all considered the integration of surface facilities with a reservoir simulator to better account for the reservoir effects over a time-lapsed simulation study. The latter employed an approximate iterative scheme using heuristics (referred to as the GLINC method) while the first two used the de-facto SQP solver.

Hepguler et al. [65] considered the variation of GLR for a model with 10 producers and 10 injectors over a 1300day production period. Kosmala et al. [66] considered a coupled reservoir and network system for well management using downhole flow control valves and also for optimal gas allocation. The implicit SQP solver in the commercial network simulator (GAP) was employed [6]. The authors note that although the cost of coupling can be high, the results are more accurate as they take account of the limits imposed by the network model over the reservoir time-steps.

Wang and Litvak [67] introduced a multi-objective condition (besides maximizing production) to minimize the lift gas rate variation between iterations using a damping factor. This had the effect of reducing the level of fluctuations encountered. The proposed method, GLINC, was compared to previous work based on a separable programming technique using a SLP formulation, using the approach of Fang and Lo [40], together with a GA and the trust region polytope based on COBYLA method (constrained optimization by linear approximation) by Powell [37]. Although the GLINC method is easy to implement and handles flow interactions directly, it does not guarantee even a locally optimal solution due to the simplified function employed during optimization and the local nature of the search. The separable programming approach does not handle flow interactions but does return the optimal solution of the SLP problem. Comparison of the methods showed that the GLINC method was the most computationally efficient.

In the paper by Wang and Litvak [67], the long-term development plan of a North Sea field comprising 21 wells was considered for both lift gas and well-rate allocation over a period of 10,000 days. The impact of the damping factor was shown in a second test case of 18 production wells with oil, water, liquid and gas-rate constraints. While not significantly impacting cumulative oil production, the addition of the damping factor significantly reduced computational cost by reducing oscillations. Computational performance measures were provided by the authors; however, as the methods differ in their implementation, these should not be considered 
conclusive. It is also worth noting that the optimization procedure is called at the first few Newton iterations of each reservoir simulation time step. There is no certainty that convergence will be completed in those steps and indeed if the Newton steps are even progressing in the right direction in that period before convergence. Hence, the optimization method and the lack of damping may not be the only cause of the oscillations encountered.

\section{Integrated Modelling Approach}

Gutierrez et al. [25] present an integrated asset approach using decline curves for the reservoir along with network and process models [68]. The integrated asset model gives a more definitive treatment of the conditions imposed by coupling in the real world. The iterative offline-online scheme proposed by Rashid [27] was utilized for gas-lift optimization. The instabilities presented by a rigorous reservoir coupling with the production network model were avoided using predictive reservoir conditions and gas-lift allocation is performed at each coupling step. A simulation comprising 10 production wells was managed over a 20 -year period for gas-lift optimization under various production constraints. These included water handling, compressor horsepower, field oil and liquid production constraints.

An integrated model comprising the reservoir, wells, surface facility model and a detailed economic model, with the purpose of optimizing the NPV of the asset, was presented by Mora et al. [69]. The model was designed to handle changes in compression, gas availability and handling capacities. The study indicated that maximum NPV does not occur when production is maximized over a common lift efficiency condition, defined as the level of production per unit gas injection over all wells, but at higher lift efficiencies. Sensitivity of the solution to oil price variation was also considered and optimization performed over a range of lift efficiencies. Results showed that depending on the price scenario selected (i.e., low to high oil price) field value is optimized by operating under different lift efficiencies. It is evident that a high oil price tends to saturate the NPV calculation. In other words, when the oil price is low, the gas and water components have a bigger impact on the NPV sensitivity to the lift efficiency employed, but if the oil price is comparatively high, these components contribute less to the NPV revenue calculation. For optimization purposes, the multi level optimization scheme from a commercial simulator (Landmark VIP) was employed [70].

Bieker et al. [71] presented the elements necessary for an offshore oil and gas production system for realtime optimization in a closed-loop. These include the acquisition, storage and processing of field data, simulationmodel management, together with optimization and control activation procedures. The intent was to devise a system that can remotely optimize production by managing facility constraints under changing operating conditions. For example, changes in pressure, lift gas availability, separator and compressor handling limits. The daily operation was managed in a fast control loop for the short-term, while a longer-term slow loop was used to account for the changing reservoir conditions. It was noted that separate optimization levels for the slow and fast loop are necessary to handle the complexity of the composite model. Generally, the application of automatic control measures enable optimal configuration settings to be implemented with minimum intervention and time delay, helping to maximize daily production. Such schemes are particularly beneficial for remote offshore locations. Bergeron et al. [14] and Mayo et al. [36] both demonstrated these concepts in practice for a single offshore well and on a field with multiple wells, respectively.

\section{Conclusion}

In an oilfield, the daily available lift gas, often constrained due to facility conditions, is prone to variation. In addition, operating conditions and handling facilities can dictate compressor deliverability and separator limits during production, while poor allocation of the available lift gas can be economically costly, leading to over-constrained or overdesigned facilities. As such, an optimal lift gas allocation is desirable to ensure that the best possible oil production or profit can be realized.

The purpose of this paper was to present a survey of the methods developed specifically to treat the continuous gaslift optimization problem. While the basic problem concerns the optimal allocation of lift gas, the broader problem can additionally include the well-rate management problem and the well strategy problem. The former concerns the control of down-hole chokes for pressure and flow-rate control, while the latter concerns the activation state or connection of wells. In some cases, the well design for gas-lift is also considered alongside the well gas-injection rate.

The problems tackled and methods employed have evolved logically, with growing computational power and confidence, stemming from single-well analysis, to lift performance based schemes, to those utilizing rigorous network solutions, all the way to full-field integrated and closedloop configurations. The methods and techniques employed cover a spectrum ranging from simple single-variable maximization to more sophisticated mixed-integer non-linear (MINLP) optimization schemes. In the intervening, SLP, SQP, DP, MILP, GA, TS, Polytope and Newton-based methods have all been applied in some manner. In addition, the equal-slope concept (including the marginal GOR approach) has led to the application of heuristic schemes and the development of solutions based on the separable and nonlinear convex nature of the lift performance curves. Clearly, some optimization methods can be applied more readily than others depending on the formulation adopted, the solver selected and the availability of derivative information from the model. Derivative-free schemes, such as GA, TS and Polytope can be applied in most settings, but suffer from a high computational overhead if the function is costly to evaluate. On the other hand, the effectiveness of gradientbased solvers is reduced if the function is non smooth or numerical derivatives are required from an expensive simulation case, as evidenced by most coupled and integrated models. Note that the use of adjoint schemes to elicit gradient 
information is possible, but will be limited to a single simulation model as sensitivity information from a coupled or integrated model must be obtained collectively, most likely, by numerical means.

Thus, while some methods are more robust and extensible to large production constrained fields comprising hundreds of wells with a great number and type of variables, others are clearly limited. Classical single-well analysis neglects the interaction of other wells in a field, curvebased models neglect the back-pressure effects imposed by connected wells and network-based simulations neglect the impact of the reservoir and the process facility model. For the latter full-field simulation, the foregoing schemes are critical to ensure speed, stability and versatility of an optimization solution in real time over the fast inner loop, while the slow outer loop can be accommodated by time-stepping the inner loop. In other words, in this case, successful control and optimization of the inner loop is a necessary prerequisite in the composite full-field integrated solution. Hence, the methods devised to ensure this must be able to handle large fields, tackle difficult and non-instantaneously flowing wells and provide accurate near-optimal solutions in a reasonable time under many operating constraints. That is, they should be able to provide solutions over the period in which a well is monitored without intervention and in which operating conditions are unlikely to change significantly. In this regard, a hierarchical optimization approach may be preferential rather than an all-in-one approach. Note that when a coupled simulation includes the reservoir model not based on decline curves, the rigor of the coupling scheme, and indeed any up scaling procedure adopted, will affect the cost of the objective function evaluation and ultimately the quality of the result achieved. However, as in any enterprise, the model should be representative of the system of interest and robust for the purpose of practical optimization.

The alternative approach to the time-stepped procedure outlined above is to optimize the entire coupled or integrated system collectively over the simulation period of interest. Due to the cost associated with a single objective function evaluation, this is achieved using derivative-free methods, still at some cost, or more practically, using proxy-based methods. Here, a fast analytical approximation model is developed from a collection of representative samples using neural network, kriging or radial basis function methods. The approximation model is then used in place of the actual simulation model in the optimization step. Iterative proxy schemes have the benefit of further reducing the number of expensive function evaluations required by sampling only in the areas of the perceived optimum at each iteration $[72,73]$. These methods have been demonstrated as robust, but are often limited to several dozen variables in dimensionality for practical reasons.

Thus, in general, the effectiveness of any approach adopted must consider the scalability of the solution method with increasing dimensionality (e.g., the objective function cost and, if required, the cost of derivative evaluation) along with the efficiency of the evaluation (e.g., the use of suitable proxy models) in order to minimize the overall computational cost. Mitigation of these factors is necessary for longer term simulation of large-scale fields for production optimization and especially when uncertainty is considered in the underlying models.

In summary, demonstration of a fully automated model, for gas-lift optimization in particular and for production optimization in general, that can handle the changing operational conditions and predict future development needs in a timely and robust manner is of value, and remains a challenging goal.

\section{Symbols}

L: Gas-lift injection rate

Q: Oil flowrate

$P_{\text {wh }}$ : Wellhead pressure

$P_{\text {bh }}$ : Bottom-hole pressure

$P_{r}$ : Reservoir pressure

$m$ : Slope value on a gas-lift performance curve.

\section{Acroynms}

BFGS: Broyden-Fletcher-Goldfarb-Shanno method BHP: $\quad$ Bottom-hole pressure

COBYLA: Constrained optimization by linear approximation

DP: $\quad$ Dynamic programming

EXIN: Method by Buitrago et al. [43]

GA: Genetic algorithm

GOAL : PipeSim gas-lift optimization module

GL: $\quad$ Gas-lift

GLIR: Gas-lift injection rate

GLINC: Method by Wang and Litvak [67]

GLPC: Gas-lift performance curve

GLR: $\quad$ Gas liquid ratio

GLV: Gas-lift valve

GOR: Gas oil ratio

IGL: Intermittent gas-lift

IGOR: Incremental GOR

IPR: Inflow performance relationship

KKT: Karush-Kuhn-Tucker conditions

MGOR: Marginal GOR

MILP: $\quad$ Mixed integer linear programming

MINLP: Mixed integer nonlinear programming

MOP: Multi-objective optimization problem

NIF: Non-instantaneous flow

NP: Non-deterministic polynomial time hard algorithm complexity

NPV: $\quad$ Net present value

NRM: $\quad$ Newton reduction method by Rashid [27]

NSGA: Non-dominated sorting GA by Deb [46]

OCI: Operating cash income

PGL: $\quad$ Plunger gas-lift

PI: $\quad$ Productivity index

PVT: $\quad$ Pressure volume and temperature

PVOCI: Preset value OCI

SLP: $\quad$ Sequential linear programming

SQP: $\quad$ Sequential quadratic programming

SOP: $\quad$ Single-objective optimization problem 
TS: Tabu search

WHP: Well-head pressure.

\section{References}

[1] K. Brown, "Overview of artificial lift systems," SPE Journal of Petroleum Technology, vol. 34, no. 10, pp. 2384-2396, 1982.

[2] N. Nishikiori, R. A. Redner, D. R. Doty, and Z. Schmidt, "Improved method for gas lift allocation optimization," in Proceedings of the SPE Annual Technical Conference \& Exhibition (ATCE '89), pp. 105-118, San Antonio, Tex, USA, October 1989.

[3] J. C. Mantecon, "Gas-lift optimisation on Barrow Island, Western Australia," in Proceedings of the SPE Asia Pacific Oil \& Gas Conference, pp. 237-246, February 1993.

[4] H. Beggs, Production Optimization Using Nodal Analysis, Oil \& Gas Consultants, 2nd edition, 2008.

[5] Schlumberger Information Solutions, PipeSim Network Simulator, Schlumberger SIS, Abingdon, UK, 2007.

[6] Petroleum Experts, GAP Network Simulator, Petroleum Experts, Edinburgh, UK, 2002.

[7] A. Bahadori, S. Ayatollahi, and M. Moshfeghian, "Simulation and optimization of continuous gas lift system in aghajari oil field," in Proceedings of the SPE Asia Pacific Improved Oil Recovery Conference, Kuala Lumpur, Malaysia, October 2001.

[8] D. Denney, "Simulation and optimization of continuous gas lift," Journal of Petroleum Technology, vol. 54, no. 5, p. 60, 2002.

[9] S. Ayatollahi, A. Bahadori, and A. Moshfeghian, "Method optimises Aghajari oil field gas lift," Oil and Gas Journal, vol. 99, no. 21, pp. 47-49, 2001.

[10] R. Vazquez-Roman and P. Palafox-Hernandez, "A new approach for continuous gas lift simulation and optimization," in Proceedings of the SPE Annual Technical Conference \& Exhibition (ATCE '05), Dallas, Tex, USA, October 2005.

[11] A. Bahadori and K. Zeidani, "Compositional model improves gas-lift optimization for Iranian oil field," Oil and Gas Journal, vol. 104, no. 5, pp. 42-47, 2006.

[12] A. Bahadori and K. Zeidani, "A new approach optimizes continuous gas lift system," World Oil, vol. 227, no. 11, pp. 4552, 2006.

[13] K. Dutta-Roy and J. Kattapuram, "New approach to gaslift allocation optimization," in Proceedings of 67th Annual Western Regional Meeting, pp. 685-691, Long Beach, Calif, USA, June 1997.

[14] T. Bergeron, A. Cooksey, and S. Reppel, "New automated continuous gas-lift system improves operational efficiency," in Proceedings of the SPE Mid-Continent Operations Symposium, Oklahoma, Okla, USA, March 1999.

[15] W. Simmons, "Optimizing continuous flow gas lift wellspart1.," Petroleum Engineer, vol. 45, no. 8, pp. 46-48, 1972.

[16] W. Simmons, "Optimizing continuous flow gas lift wellspart2," Petroleum Engineer, vol. 44, no. 10, pp. 68-72, 1972.

[17] E. P. Kanu, J. Mach, and K. E. Brown, "Economic approach to oil production and gas allocation in continuous gas-lift," Journal of Petroleum Technology, vol. 33, no. 10, pp. 1887-1892, 1981.

[18] T. Mayhill, "Simplified method for gas-lift well problem identification and diagnosis," in Proceedings of the SPE Fall AIME Meeting, Dallas, Tex, USA, October 1974.

[19] G. A. Alarcón, C. F. Torres, and L. E. Gómez, "Global optimization of gas allocation to a group of wells in artificial lift using nonlinear constrained programming," Journal of Energy Resources Technology, Transactions of the ASME, vol. 124, no. 4, pp. 262-268, 2002.

[20] J. Redden, T. Sherman, and J. Blann, "Optimizing gas-lift systems," in Proceedings of the SPE Fall AIME Meeting, pp. 113, Dallas, Tex, USA, October 1974.

[21] J. Clegg, "Discussion of economic approach to oil production and gas allocation in continuous gas lift," SPE Journal of Petroleum Technology, pp. 310-302, 1982.

[22] E. P. Kanu, "Author's reply to discussion of economic approach to oil production and gas allocation in continuous gas lift," SPE Journal of Petroleum Technology, p. 519, 1982.

[23] R. Stoisits, P. Scherer, and S. Schmidt, “. Gas optimization at the kuparak river field," in Proceedings of the SPE Annual Technical Conference \& Exhibition (ATCE'94), pp. 35-42, New Orleans, La, USA, September 1994.

[24] J. L. Weiss, W. H. Masino, G. P. Starley, and J. D. Bolling, "Large-scale facility expansion evaluations at the Kuparuk river field," in Proceedings SPE Regional Meeting, pp. 297-304, Bakersfield, Calif, USA, April 1990.

[25] F. Gutierrez, A. Hallquist, M. Shippen, and K. Rashid, "A new approach to gas lift optimization using an integrated asset model," in SPE International Petroleum Technology Conference (IPTC '07), pp. 1371-1380, Dubai, UAE, December 2007.

[26] K. Rashid, "A method for gas lift optimization," in Proceedings of the SIAM Annual Meeting, Boston, Mass, USA, May 2008.

[27] K. Rashid, "Optimal allocation procedure for gas-lift optimization," Industrial and Engineering Chemistry Research, vol. 49, no. 5, pp. 2286-2294, 2010.

[28] Z. Schmidt, D. R. Doty, B. Agena, T. Liao, and K. E. Brown, "New developments to improve continuous-flow gas lift utilizing personal computers," in Proceedings of the SPE Annual Technical Conference \& Exhibition (ATCE '90), pp. 615-630, New Orleans, La, USA, September 1990.

[29] Y. Chia and S. Hussain, "Gas lift optimization efforts and challenges," in Proceedings of the SPE Asia Improved Oil Recovery Conference, pp. 2-9, Kuala Lumpur, Malaysia, October 1999.

[30] Schlumberger Information Solutions, Eclipse Reference Manual, Schlumberger SIS, Abingdon, UK, 2006.

[31] T. A. Everitt, "Gas-lift optimization in a large, mature GOM field," in Proceedings of the SPE Annual Technical Conference \& Exhibition (ATCE '94), pp. 25-33, New Orleans, La, USA, September 1994.

[32] R. Edwards, D. L. Marshall, and K. C. Wade, "Gas-lift optimization and allocation model for manifolded subsea wells," in Proceedings of the European Petroleum Conference (EUROPEC '90), pp. 535-545, The Hague, The Netherlands, October 1990.

[33] A. A. Ferrer and R. Maggiolo, "Use of a computerized model in the optimization of continuous gas-lift operations," in Proceedings of the SPE Production Operations Symposium, pp. 87-96, April 1991.

[34] Schlumberger Information Solutions, ,GOAL Gas-Lift Optimizer, Schlumberger SIS, Abingdon, UK, 1998.

[35] R. F. Stoisits, E. C. Batesole, J. H. Champion, and D. H. Park, "Application of nonlinear adaptive modeling for rigorous representation of production facilities in reservoir simulation," in Proceedings of the SPE Annual Technical Conference \& Exhibition (ATCE '92), pp. 425-434, Washington, DC, USA, October 1992.

[36] O. Mayo, F. Blanco, and J. Alvarado, "Procedure optimizes lift gas allocation," Oil \& Gas Journal, vol. 97, no. 13, pp. 38-41, 1999. 
[37] P. Gill, W. Murray, and M. Wright, Practical Optimization, Academic Press, 12th edition, 2000.

[38] R. Fletcher, Practical Methods of Optimization, John Wiley \& Sons, 2nd edition, 2000.

[39] K. K. Lo, Optimum Lift-Gas Allocations under Multiple Production Constraints, Society of Petroleum Engineers, 1992.

[40] W. Y. Fang and K. K. Lo, "A generalized well-management scheme for reservoir simulation," SPE Reservoir Engineering, vol. 11, no. 2, pp. 116-120, 1996.

[41] S. Handley-Schachler, C. McKie, and N. Quintero, "New mathematical techniques for the optimization of oil \& gas production systems," in Proceedings of the SPE European Petroleum Conference (EUROPEC '00), pp. 429-436, October 2000.

[42] R. Salazar-Mendoza, "New representative curves for gas lift optimization," in Proceedings of the SPE 1st International Oil Conference, Cancun, Mexico, August 2006.

[43] S. Buitrago, E. Rodriguez, and D. Espin, "Global optimization techniques in gas allocation for continuous flow gas lift systems," in Proceedings of the SPE Gas Technology Symposium, pp. 375-383, Calgary, Canada, May 1996.

[44] T. Ray and R. Sarker, "Multiobjective evolutionary approach to the solution of gas lift optimization problems," in Proceedings of the IEEE Congress on Evolutionary Computation (CEC '06), pp. 3182-3188, British Columbia, Canada, July 2006.

[45] E. R. Martinez, W. J. Moreno, J. A. Moreno, and R. Maggiolo, "Application of genetic algorithm on the distribution of gas lift injection," in Proceedings of the 3rd SPE Latin American and Caribbean Petroleum Engineering Conference, pp. 811-818, Buenos Aires, Argentina, April 1994.

[46] K. Deb, Multi-Objective Optimization Using Evolutionary Algorithms, John Wiley \& Sons, 2001.

[47] K. Dutta-Roy, S. Barua, and A. Heiba, "Computer-aided gas field planning and optimization," in Proceedings of the SPE Production Operations Symposium, pp. 511-515, Oklahoma, Okla, USA, March 1997.

[48] M. S. Nadar, T. S. Schneider, K. L. Jackson, C. J. N. McKie, and J. Hamid, "Implementation of a total-system productionoptimization model in a complex gas lifted offshore operation," SPE Production and Operations, vol. 23, no. 1, pp. 5-13, 2008.

[49] M. Vazquez, A. Suarez, H. Aponte, L. Ocanto, and J. Fernandes, "Global optimization of oil production systems, a unified operational view," in Proceedings of the SPE Annual Technical Conference \& Exhibition (ATCE '01), New Orleans, La, USA, October 2001.

[50] R. Stoisits, D. MacAllister, M. McCormack, A. Lawal, and D. Ogbe, "Production optimization at the kuparak river field utilizing neural networks and genetic algorithms," in Proceedings of the SPE Mid-Continent Operations Symposium, Oklahoma, Okla, USA, March 1999.

[51] S. Moitra, S. Chand, S. Barua, D. Adenusi, and V. Agrawal, "A field-wide integrated production model and asset management system for the mumbai high field," in Proceedings of the SPE Offshore Technology Conference, Houston, Tex, USA, April 2007.

[52] K. Rashid, S. Demirel, and B. Couët, "Gas-lift optimization with choke control using a mixed-integer nonlinear formulation," Industrial and Engineering Chemistry Research, vol. 50, no. 5, pp. 2971-2980, 2011.

[53] V. D. Kosmidis, J. D. Perkins, and E. N. Pistikopoulos, "Optimization of well oil rate allocations in petroleum fields,"
Industrial and Engineering Chemistry Research, vol. 43, no. 14, pp. 3513-3527, 2004.

[54] D. W. Peaceman, "Interpretation of well-block pressures in numerical reservoir simulation," Society of Petroleum Engineers, vol. 18, no. 3, pp. 183-194, 1978.

[55] R. Sachdeva, Z. Schmidt, J. P. Brill, and R. M. Blais, "Two pahse flow through chokes," in SPE Annual Technical Conference and Exhibition, Society of Petroleum Engineers, New Orleans, Louisiana, 5-8 October 1986.

[56] ILOG, ILOG CPLEX User's Manual, ILOG, 2002.

[57] E. Camponogara and P. H. R. Nakashima, "Solving a gas-lift optimization problem by dynamic programming," European Journal of Operational Research, vol. 174, no. 2, pp. 1220-1246, 2006.

[58] V. D. Kosmidis, J. D. Perkins, and E. N. Pistikopoulos, "A mixed integer optimization formulation for the well scheduling problem on petroleum fields," Computers and Chemical Engineering, vol. 29, no. 7, pp. 1523-1541, 2005.

[59] J. E. Davidson and B. Beckner, "Integrated optimization for rate allocation in reservoir simulation," in SPE Reservoir Simulation Symposium, Houston, Tex, USA, February 2003.

[60] P. Wang, M. Litvak, and K. Aziz, "Optimization of production operations in petroleum fields.," in Proceedings of the SPE Annual Technical Conference \& Exhibition (ATCE '02), San Antonio, Tex, USA, September 2002.

[61] H. Fujii and R. Horne, "Multivariate optimization of networked production systems," SPE Production \& Facilities, vol. 10, no. 3, pp. 165-171, 1995.

[62] W. Press, S. Teukolsky, W. Vetterling, and B. Flannery, Numerical Recipes in $\mathrm{C}++$, Cambridge University Press, Cambridge, UK, 3rd edition, 2002.

[63] J. Carroll and R. Horne, "Multivariate optimization of production systems," SPE Journal of Petroleum Technology, vol. 44, no. 7, pp. 782-789, 1992.

[64] M. R. Palke and R. N. Horne, "Nonlinear optimization of well production considering gas lift and phase behavior," in Proceedings of the SPE Production Operations Symposium, pp. 341-356, Oklahoma, Okla, USA, March 1997.

[65] G. G. Hepguler, K. Dutta-Roy, and W. A. Bard, "Integration of a field surface and production network with a reservoir simulator," SPE Computer Applications, vol. 12, no. 4, pp. 8892, 1997.

[66] A. Kosmala, S. I. Aanonsen, A. Gajraj et al., "Coupling of a surface network with reservoir simulation," in Proceedings of the SPE Annual Technical Conference \& Exhibition (ATCE '03), pp. 1477-1487, Denver, Colo, USA, October 2003.

[67] P. Wang and M. Litvak, "Gas lift optimization for longterm reservoir simulations," SPE Reservoir Evaluation and Engineering, vol. 11, no. 1, pp. 147-153, 2008.

[68] Schlumberger Information Solutions, Avocet-IAM Integrated Asset Modeller, Schlumberger SIS, Calgary, Canada, 2007.

[69] O. Mora, R. A. Startzman, and L. Saputelli, "Maximizing net present value in mature gas-lift fields," in Proceedings of the SPE Hydrocarbon Economics and Evaluation Symposium: Hydrocarbon Development-A Global Challenge, pp. 123-131, Dallas, Tex, USA, April 2005.

[70] Landmark Graphics Corporation, VIP Simulator, Landmark Graphics Corporation, 2003.

[71] H. P. Bieker, O. Slupphaug, and T. A. Johansen, "Real-time production optimization of oil and gas production systems: a technology survey," SPE Production and Operations, vol. 22, no. 4, pp. 382-391, 2007. 
[72] B. Couët, H. Djikpesse, D. Wilkinson, and T. Tonkin, "Production enhancement through integrated asset modeling optimization," in SPE Production and Operations Conference and Exhibition (POCE '10), pp. 375-384, Tunis, Tunisia, June 2010.

[73] K. Rashid, S. Ambani, and E. Cetinkaya, "An adaptive multiquadric radial basis function method for expensive black-box mixed-integer nonlinear constrained optimization," Engineering Optimization. In press. 

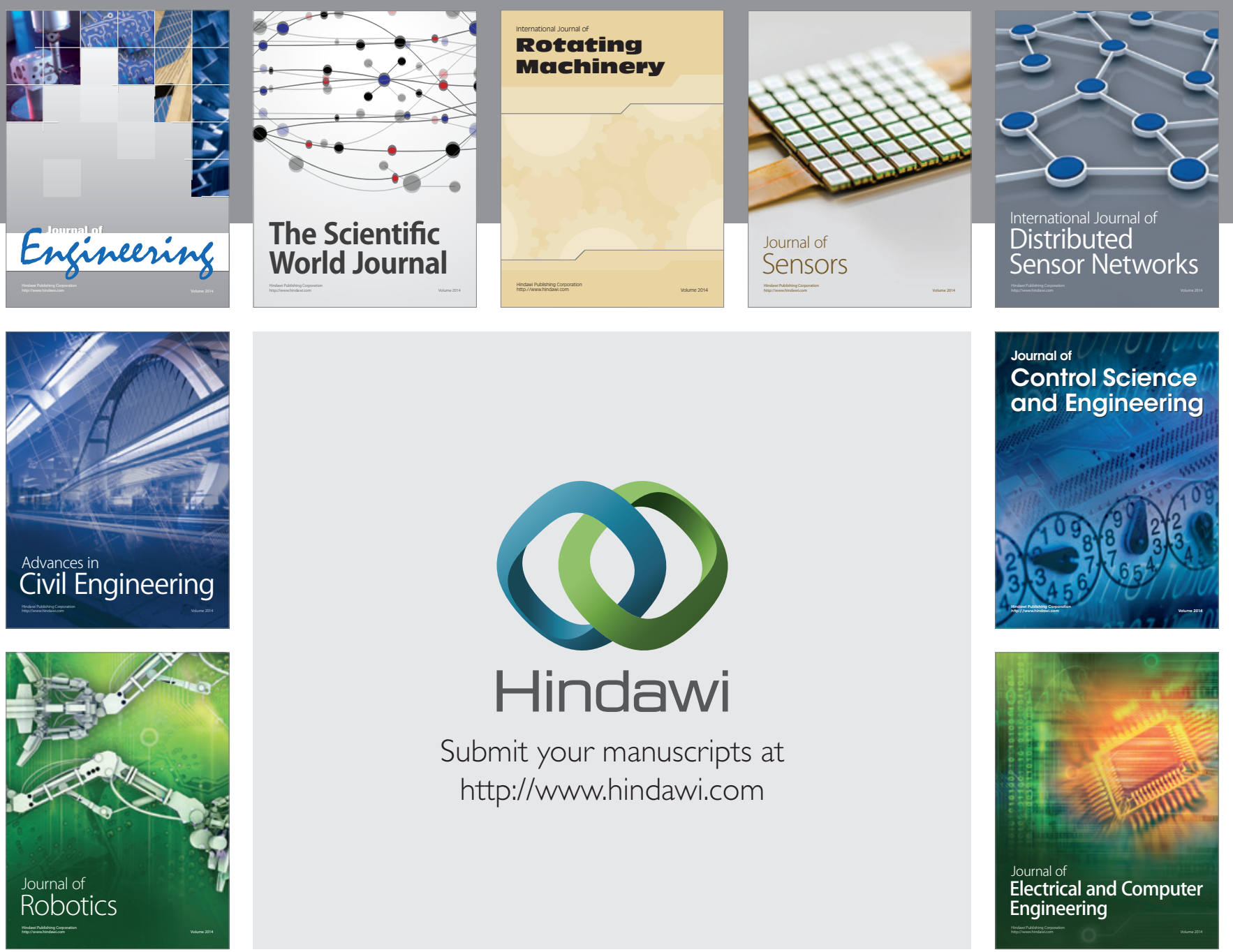

Submit your manuscripts at

http://www.hindawi.com
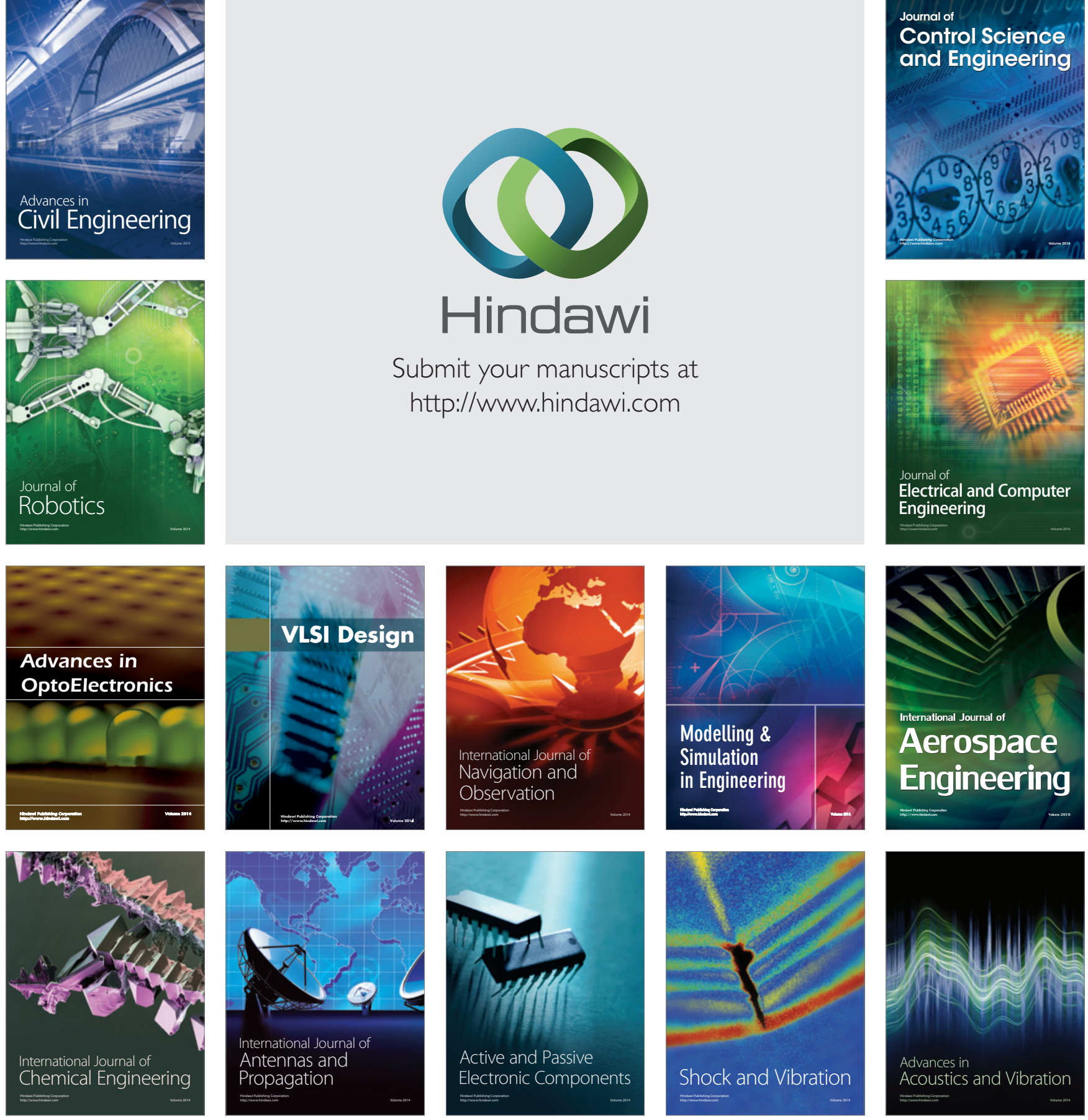